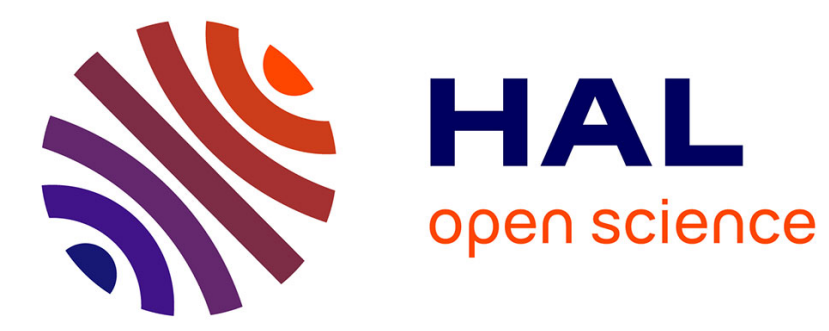

\title{
Croissance et renoncements : vieillir à l'épreuve du temps
}

\author{
Jean-François Toussaint, Bernard Swynghedauw
}

\section{To cite this version:}

Jean-François Toussaint, Bernard Swynghedauw. Croissance et renoncements : vieillir à l'épreuve du temps. Revue Esprit, 2010. hal-02058946

\section{HAL Id: hal-02058946 https://hal-insep.archives-ouvertes.fr/hal-02058946}

Submitted on 6 Mar 2019

HAL is a multi-disciplinary open access archive for the deposit and dissemination of scientific research documents, whether they are published or not. The documents may come from teaching and research institutions in France or abroad, or from public or private research centers.
L'archive ouverte pluridisciplinaire HAL, est destinée au dépôt et à la diffusion de documents scientifiques de niveau recherche, publiés ou non, émanant des établissements d'enseignement et de recherche français ou étrangers, des laboratoires publics ou privés. 


\section{Croissance et renoncements : vieillir à l'épreuve du temps}

Jean-François Toussaint, Université Paris Descartes, Directeur de I'Irmes

Bernard Swynghedauw, Directeur de Recherche, U942 Inserm, Lariboisière

Article publié dans la revue Esprit, juillet 2010

bien vivre et bien mourir constituent un seul et même exercice mais vieillir, ô vieillir...

Épicure

Jacques Brel

L'Homme écrit sa trajectoire avec les lettres du vivant. Il en respecte la complexité, les contraintes et les lois : dépendance énergétique, quête d'efficience, cycles, transmission, disparition. Son parcours individuel s'inscrit entre deux instants, conception et mort, entre lesquels se tendent les deux cordes de la croissance et du déclin. La première résume la puissance vitale expansive et culmine au moment du cycle reproductif. La seconde imprime sa marque sur tous les systèmes de notre univers connu.

Le vieillissement peut donc être aujourd'hui considéré comme le processus incarnant la flèche du temps ${ }^{1}$ et son inéluctable impact sur les structures du vivant. Il révèle I'instabilité des éléments qui nous constituent autant que la fragilité des mécanismes de réparation ${ }^{2}$. Il permet aussi de comprendre l'adéquation des échelles d'espace et de temps qui régissent la vie des cellules, des individus et des espèces : une souris vit environ 3 ans avec une fréquence cardiaque de 600 battements par minute. Celle-ci lui autorise un milliard de pulsations environ tout au long de sa vie, un nombre très constant pour l'ensemble des mammifères, puisqu'à l'autre bout du spectre, une baleine à bosse peut vivre jusqu'à 90 ans avec une fréquence cardiaque inférieure à 20 battements par minute.

Vieillir se résume à une dégradation exponentielle, qui, pour chacun d'entre nous, débute dès la conception, s'amplifie lentement pour dépasser les capacités de croissance à l'âge de 25 ans. Ce point d'intersection des courbes de progression et de décroissance signe notre optimum reproductif : c'est l'âge auquel les femmes ont le risque de mortalité le plus faible à l'accouchement ${ }^{3}$, et le moment où la transmission des aptitudes à la génération suivante permet de contourner le processus de dégradation individuel.

Les capacités d'une espèce sont à tout moment le résultat de l'expression de ses gènes dans le cadre autorisé par son environnement ${ }^{4}$. Si I'Homme a développé d'extraordinaires possibilités d'agir sur celui-ci, à tel point qu'il en perturbe désormais les grands cycles régulateurs ${ }^{5}$, il a bénéficié

\footnotetext{
${ }^{1}$ S. W. Hawking, Une brève histoire du temps. Du big bang aux trous noirs, Paris, Flammarion, 1989.

I Prigogine, I Stengers. Entre le temps et l'éternité. Paris, Fayard, 1988

${ }^{2}$ L Hayflick. How and Why We Age. New York, Ballantine Books, 1994

${ }^{3}$ Rapport du Comité national d'experts sur la mortalité maternelle 2001-2006. InVS, Bulletin épidémiologique hebdomadaire (BEH) $n^{\circ}$ 2-3 du 19 janvier 2010

${ }^{4}$ B Swynghedauw. Quand le gène est en conflit avec son environnement. Bruxelles, De Boeck, 2009.

${ }^{5}$ ce que Paul Crützen résume sous le terme d'Anthropocène
} 
de conditions qui l'ont amené à établir ses performances d'aujourd'hui (diminution de la mortalité d'origine infectieuse et de la mortalité infantile, accroissement de sa taille et de son poids, lutte contre les maladies liées à l'âge : athérosclérose, cancers, démences...) et repousser la mort aux portes de la neuvième décennie alors qu'elle se présentait souvent dès la troisième. Ces progrès depuis deux siècles conduisent à ce que l'on peut qualifier de remarquable «expansion phénotypique » mais ils ne peuvent résulter, sur un temps si court (environ 10 générations), de modifications de la structure du génome.

\section{Limites}

La question qui se pose est désormais celle de nos frontières biologiques. Jusqu'où vieillirons-nous ? Homo Habilis atteint-il les limites de son espérance de vie alors que sa technologie semblait pouvoir les repousser encore ? L'accumulation des contraintes qui pèsent sur lui n'est-elle pas amenée à dépasser ses capacités d'adaptation ?

Il semble qu'apparaissent en effet les premiers signes d'un net ralentissement. Nombre de processus biologiques sont marqués par une évolution asymptotique aboutissant à un plafond d'aptitude : la démonstration récente des limites de nos fonctions spécifiquement humaines (vitesse maximale de déplacement à la course, à la nage, à vélo, longueur et hauteurs des sauts, poids soulevés ou lancés, vitesse de nos bateaux à rame ou à voile...) évoque un arrêt de notre progression d'ici une à deux générations (Figure 1).

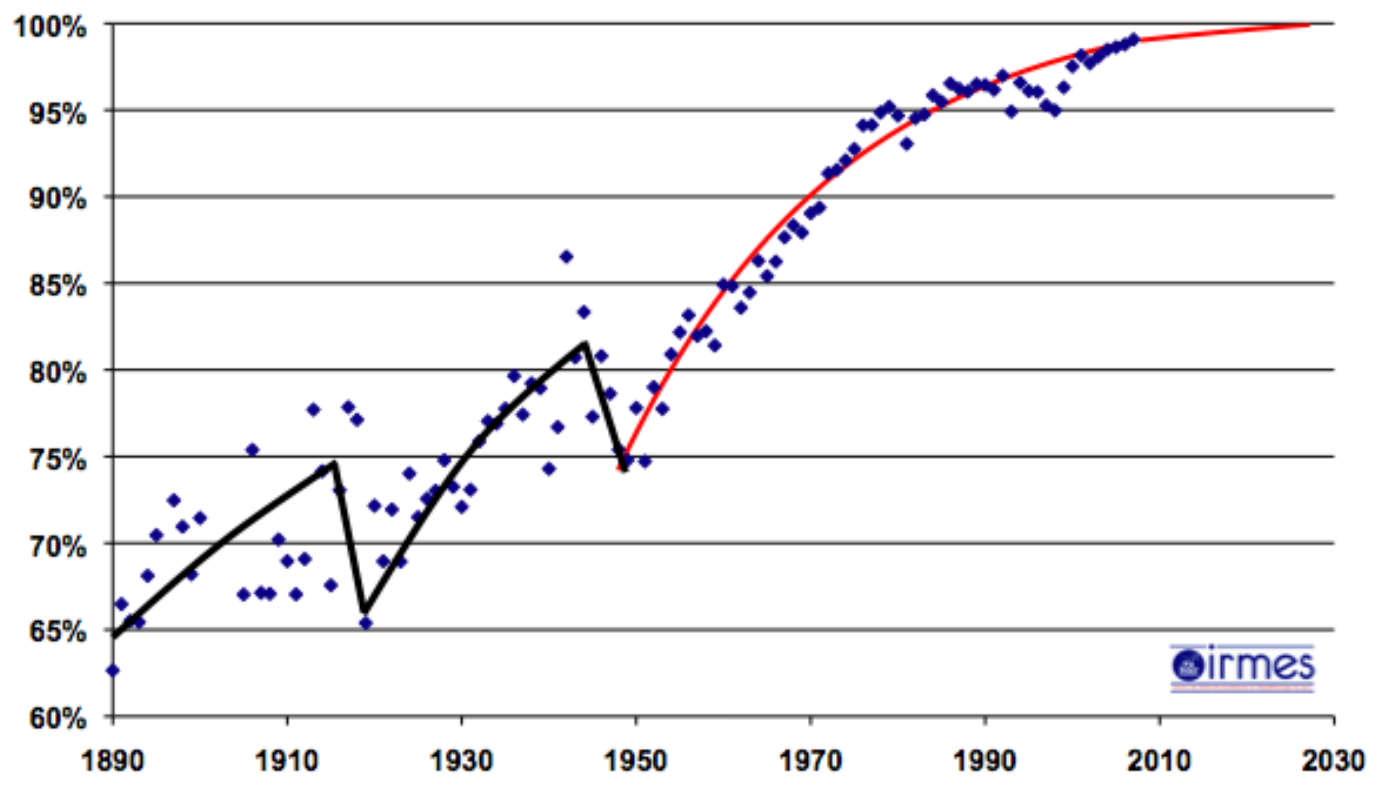

Figure 1: Progression des 3260 records du monde officiels dans 148 disciplines olympiques. Après un recul important lors de chaque guerre mondiale, la dernière asymptote montre que dans 20 à 30 ans, les maxima physiologiques seront probablement atteints (G Berthelot. "The Citius End", PLOS ONE, 2008, 3, e1552). 
Mais pour étudier les 6,8 milliards d'individus que compte notre espèce, et leurs comportements, il faut changer de perspective et d'outil analytique. À la base de l'épidémiologie, cette mathématique des grands nombres possède l'étonnante capacité de décrire les évolutions de nos principaux groupes sociaux, nationaux, ethniques ou culturels. Pour les valeurs maximales (record de Jeanne Calment) comme pour les valeurs médianes (espérance de vie moyenne des Français), cette propriété ne manque d'ailleurs pas d'interroger sur l'avenir proche ${ }^{6}$ et sa prédictibilité.

Les observateurs du vieillissement se partagent comme toujours entre réalistes et optimistes, c'est-à-dire sur un système d'interprétation subjectif des faits observés et, au fond, sur des conceptions et des croyances. Les arguments développés par les derniers laissent présager encore quelques avancées dans les pays développés - d'ordre technologique ou organisationnel - ou dans les pays en développement, à condition que se réalise un vrai partage des ressources et des connaissances. Mais de nombreux articles depuis 20 ans ont poursuivi I'utopie de la prospective linéaire, à savoir la continuation des courbes constatées dans la deuxième moitié du XXème siècle. Or cette erreur conceptuelle s'est déjà révélée dans la justification fallacieuse de records sportifs illimités ${ }^{7}$. Elle apparaît maintenant comme emblématique de notre incapacité à imaginer la fin de notre optimisation et à anticiper le début de nos soucis.

\section{Principe de croissance}

Dans l'espèce humaine, comme dans de nombreuses autres, les courbes phénotypiques en fonction de l'âge relient la puissance maximale (qui culmine vers 25 ans pour la vitesse de course) et la durée de vie la plus longue (Figure 2). Autrement dit les capacités les plus élevées sont intimement liées à la survie la plus longue. De fait, les critères de dépendance des personnes en fin de vie sont inversement proportionnels à leur degré d'autonomie et à la force de leur quadriceps ${ }^{8}$, qui conditionne autant la qualité de vie et leurs possibilités de déplacements que la durée de séjour dans les maisons de retraite.

\footnotetext{
${ }^{6} \mathrm{E}$ Le Bourg. Le vieillissement en questions. CNRS Éditions, HC Sciences

7 AJ Tatem AJ. "Athletics: Momentous sprint at the 2156 Olympics ?", Nature, 2004, 431, p. 525

${ }^{8}$ ME Cress. "Maximal voluntary and functional performance levels needed for independence in adults aged 65 to 97 years", Phys Ther, 2003, 83, p. 37
} 


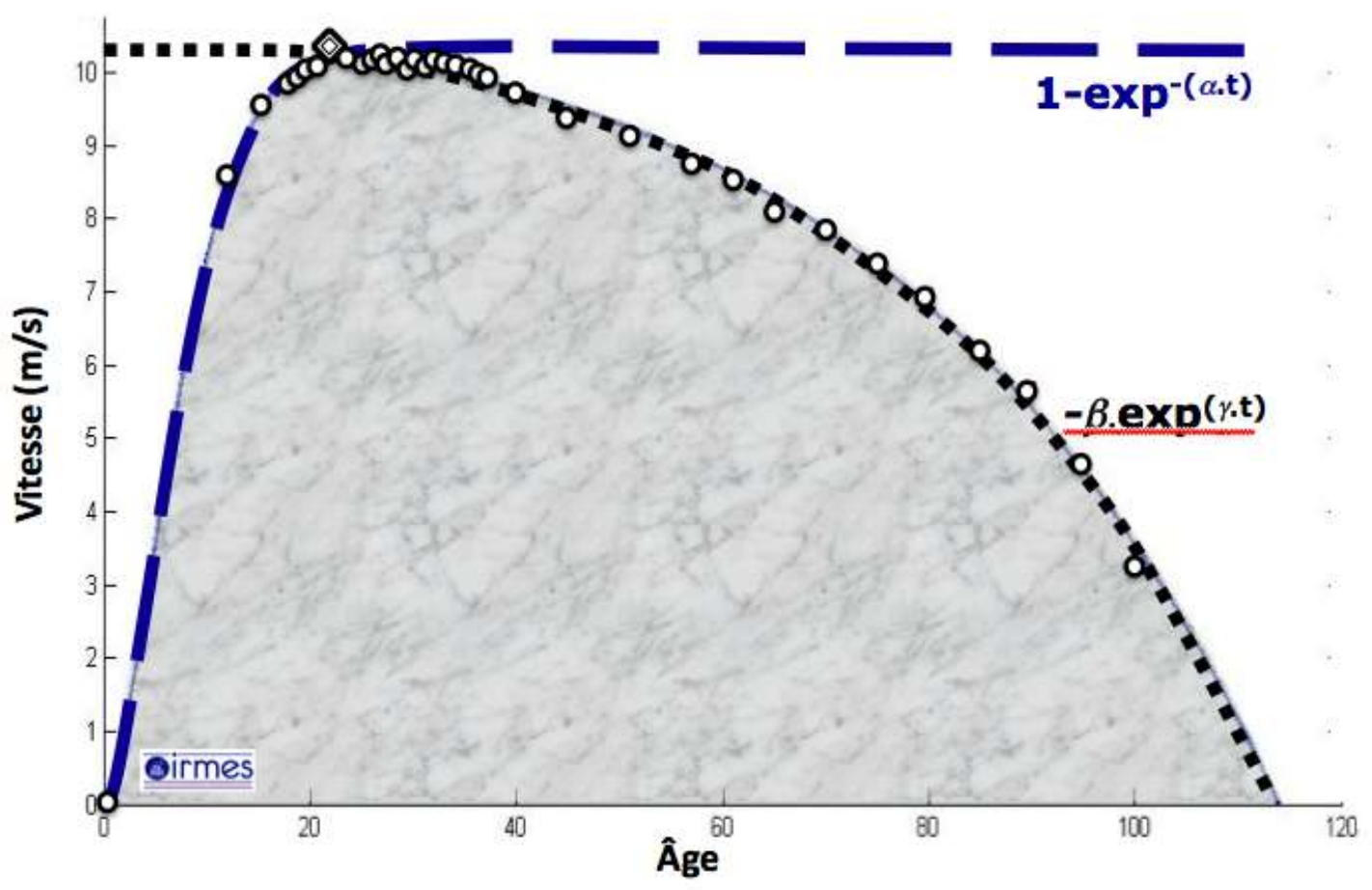

Figure 2 : Records du Monde (vitesse de course maximale) selon l'âge sur le $100 \mathrm{~m}$ masculin (chaque point est issu des données de I'IAAF, fédération internationale d'athlétisme, incluant ses compétitions vétérans, les Master Games). Cette courbe

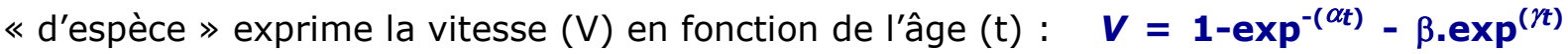
$\left(r^{2}=0,99\right)$. Elle associe deux exponentielles : la première est croissante en 1 -exp ${ }^{-(\alpha t)}$ (longs traits discontinus) et la seconde est décroissante en $-\beta \cdot \exp ^{(\gamma t)}$ (pointillés). La surface sous la courbe (marbrée) représente le potentiel maximal d'espèce. Avec un coefficient de détermination $\left(r^{2}\right)$ très proche de 1 , l'adéquation du modèle aux données réelles est extrêmement forte. Usain Bolt, le point le plus élevé à 23 ans (losange), est actuellement à la croisée des deux courbes. Individuellement, bien qu'à un niveau inférieur, nous suivons tous le même type de parcours suivant en cela une propriété fractale remarquable : I'autosimilarité de la fonction qui relie l'énergie, la croissance, l'apogée (au phénotype maximal), I'altération et la disparition (S Len, Progressions individuelles de la performance : un modèle unifié. $4^{\text {ème }}$ Symposium de l'Irmes, 2009).

Cette double relation ornemente d'une étrange mathématique bleue ${ }^{9}$ le très antique débat des puissances qui nous gouvernent; celles qui, d'Osiris à Faust, règleraient notre destin et que Freud a réactualisées dans le combat d'Eros et de Thanatos. Les pulsions de croissance, propre du vivant, et de mort - propre du temps - s'illustrent ici dans une équation sommaire, duale et irréductible. On comprend dès lors qu'elles sont loin de ne concerner que le versant psychologique de notre personnalité mais qu'elles sont probablement l'une des bases biologiques de l'existence.

Cette relation accompagne aussi le déclin cognitif: la plupart des capacités de mémoire (épisodique de court-terme, de long-terme,

\footnotetext{
9 Léo Ferré. La mémoire et la mer (poème), 1970 (... Et je voyais ce qu'on pressent / Quand on pressent l'entrevoyure / Entre les persiennes du sang / Et que les globules figurent / Une mathématique bleue / Dans cette mer jamais étale / D'où nous remonte peu à peu / Cette mémoire des étoiles).
} 
visuelle, mémoire de travail) ou de raisonnement décroissent de façon similaire avec le temps ${ }^{10}$. Ces capacités restent très corrélées tout au long de la vie: les performances initiales expliquent une très grande part des aptitudes cognitives et mémorielles en fin de $v^{1} e^{11}$, inscrivant une cohérence individuelle remarquable, évoquée par la double exponentielle.

Le potentiel de chacun - ses capacités physiques et mentales maximales possibles (mais non son parcours réel) - est donc en théorie mesurable sur la pente à l'origine de cette courbe, une «mal-mesure » de l'Homme chère à Stephen Jay Gould. Cette inclinaison initiale se révèle comme I'un des premiers grands indicateurs et la surveillance des conditions de grossesse remplit cet objectif visant à limiter les déficits précoces autant que tardifs. Les travaux de DJ Barker ${ }^{12}$ ont en effet montré à quel point les premiers moments sont primordiaux et permettent d'anticiper les conditions d'altération finale comme les risques d'affections dégénératives.

Il faut donc singulariser les mécanismes de la sénescence qui modulent la longévité et exposent les organismes aux maladies, séquence inévitablement conclue par l'arrêt thermodynamique : la mort de l'individu, privé d'énergie, de connexions, de chaleur et de mouvement. Dans la période de développement économique des deux derniers siècles, tous les indicateurs de croissance ont accentué notre penchant naturel visant à nier cette échéance. Les sociétés humaines ont alors confié à la recherche biologique et médicale le soin d'imaginer des solutions à notre quête d'éternité. Mais le paradoxe devient insupportable, lorsque la science démontre désormais que la fin de chaque cycle individuel est indissociable de la survie de l'espèce ${ }^{13}$.

\section{Biologie du vieillissement}

L'augmentation actuelle de la durée de vie est due à I'activité humaine ; elle est à la fois récente et unique dans l'évolution. Pour Sapiens, dans les conditions du début de ce siècle, la durée de vie moyenne est de 68 ans et s'élève à 80 ans dans les pays les plus développés (Source OMS). Elle n'a pourtant été en moyenne que d'environ 30 ans pour les 100 milliards d'individus qui se sont succédés durant les 200 millénaires de notre existence. Et l'espérance de vie en Afrique sub-Saharienne ne dépasse d'ailleurs qu'à peine la quarantaine : vieillir longtemps reste le privilège des populations riches des climats tempérés.

\footnotetext{
${ }_{10}^{10}$ DC Park. "The adaptive brain: aging and neurocognitive scaffolding", Annual Review Psychology, 2009, 60, p. 173

${ }^{11}$ IJ Deary. "Stability of differences in mental ability from childhood to old age", Intelligence, 2000, 28, p. 49

12 En étudiant les conséquences de la famine de I'hiver 1944-45 à Amsterdam, il est le premier à montrer le rôle majeur de la nutrition fœtale sur les maladies liées au vieillissement ("The fetal and infant origins of adult disease", British Medical Journal, 1990, 301, p. 1111).

${ }^{13}$ Jacques Ruffié. Le Sexe et la mort. Paris, Odile Jacob, 1986
} 
L'organisation du génome chez tous les êtres vivants détermine en grande partie la durée de vie moyenne des individus d'une espèce ( 4 ans pour la gerboise, plus de 2000 ans pour le séquoia) mais les contraintes environnementales modulent largement cette valeur pour chaque individu. À contrainte forte, moyenne de vie brève. On a longtemps cherché une cause simple, un gène de la longévité ou le déclin d'une fonction essentielle, sur lequel on puisse agir. Cette manière de voir est maintenant abandonnée même si certains chercheurs ou certaine presse, reflet de nos illusions, continuent de I'annoncer régulièrement. Vieillir est aussi compliqué que vivre. Ce processus complexe et multifactoriel, n'est, en revanche, pas programmé.

\section{Évolution}

En biologie rien n'est vrai qui ne soit en accord avec l'évolution ${ }^{14}$; le vieillissement n'échappe pas à cette règle. L'évolution procède à la fois par hasard et par pression sélective : une mutation survenue au hasard qui donne à son porteur un avantage sera conservée; à l'inverse les mutations délétères disparaissent avec le temps. Cela suppose néanmoins que le porteur soit en âge de procréer. Si un gène a des effets négatifs dès l'enfance, il réduit la possibilité de son porteur de se reproduire et de transmettre ce gène à la génération suivante. Il est donc rapidement éliminé.

Si par contre ce gène n'a d'effets négatifs qu'après l'âge de la reproduction, il peut être conservé. Le vieillissement et la réduction de l'espérance de vie pourraient résulter de l'accumulation de ces gènes ou mutations délétères à expression tardive. Il pourrait aussi provenir de la ré-expression de gènes utiles à la croissance chez l'enfant, mais délétères au delà (concept de pléiotropie antagoniste) ${ }^{15}$ comme le montre le gène responsable de l'ostéogenèse chez l'enfant, qui renforce ses structures osseuses mais provoque aussi les calcifications valvulaires du sujet âgé.

Cette vue évolutionniste ${ }^{16}$ se heurte néanmoins à quelques évidences : le vieillissement que nous connaissons de nos jours est d'un type jamais expérimenté dans l'histoire. Le rôle de l'environnement est capital pour en décrire les modulations et explique pourquoi la sénescence fait maintenant le lit des pathologies liées à l'âge. Cancers et maladies cardiovasculaires ont, au cours de cette transition épidémiologique, remplacé les maladies infectieuses comme première cause de mortalité, dans un constant déplacement d'équilibres souligné par Georges Canguilhem ${ }^{17}$.

\section{Sénescence physiologique}

\footnotetext{
14 Theodosius Dobzhansky (1900 - 1975)

${ }^{15}$ B Swynghedauw. Op cité

${ }^{16}$ MR Rose. The long tomorrow. How advances in evolutionary biology can help us postpone aging, New York, Oxford University Press, 2005

${ }^{17}$ G Canguilhem. Le Normal et le Pathologique. (1943), Paris, PUF, 1966
} 
Si notre compréhension des phénomènes concernant les tissus et l'organisme dans sa globalité n'en est qu'à ses débuts, les mécanismes par lesquels une cellule isolée vieillit sont en partie élucidés et la sénescence réplicative, qui qualifie ce processus, bien documentée. Les cellules en culture ne peuvent en effet se multiplier à l'infini et perdent aussi leur capacité de se diviser in vivo. Cette forme de sénescence concerne également les cellules souches ${ }^{18}$. D'autres formes de vieillissement tissulaire, comme la fibrose (dépôt de tissu conjonctif non fonctionnel), s'ajoutent à ce mécanisme.

La production des protéines s'accompagne aussi d'une destruction et leur synthèse ne se fait pas sans lyse. L'immobilisation et la sédentarité sont par exemple à l'origine de l'activation de systèmes lytiques et d'autophagie («qui se mange soi-même»). De fait, lyse et fibrose finissent par l'emporter: I'évolution a conféré à notre organisme une importante faculté de réparation des molécules déficientes, jusqu'à la maturité. Mais dès l'instant de cet optimum reproductif franchi, ces capacités de régénération semblent dépassées. Quatre grandes causes soulignent actuellement les mécanismes du vieillissement : la place des télomères, le rôle de l'épigénétique, l'instabilité de I'ADN et l'effet sur la circulation seront successivement revus avant d'envisager les deux principaux mécanismes préventifs.

\section{Télomères et épigénétique}

Le prix Nobel de Médecine et de Physiologie 2009 a mis en lumière les mécanismes de protection des chromosomes durant les divisions cellulaires ${ }^{19}$. Assurée par des structures répétitives, les télomères, cette fonction est maintenue par une enzyme, la télomérase, très active durant toutes les phases de croissance.

À chaque division quelques fragments du télomère sont néanmoins perdus et l'activité de l'enzyme, qui décroît avec le temps, devient insuffisante pour compenser intégralement ce déficit. Le télomère se réduit donc et sa longueur devient un marqueur du vieillissement chromosomique, prédictif de la mortalité après 60 ans $^{20}$. Le raccourcissement des télomères est I'un des facteurs responsables de la sénescence réplicative : sans télomère, plus de division cellulaire.

\footnotetext{
${ }^{18}$ RB Effros. "Hematopoietic cells and replicative senescence", Exp Gerontol. 2002, 37, p. 191

19 EH Blackburn ("Switching and signaling at the telomere", Cell, 2001, 106, p. 661), CW Greider ("Identification of a specific telomere terminal transferase activity in Tetrahymena extracts", Cell, 1985, 43, p. 405), JW Szostak ("Cloning yeast telomeres on linear plasmid vectors", Cell, 1982, 29, p. 245). Ce mécanisme, qui prévient la fusion des chromosomes entre eux, est très ancien. Il existe chez les levures, les ciliés et chez I'homme.

${ }^{20}$ RM Cawthon. "Association between telomere length in blood and mortality in people aged 60 years or older", Lancet, 2003, 361, p. 393
} 
Il semble que certains déterminants du vieillissement puissent également agir sur la structure globale du vivant. La recherche en la matière n'a longtemps été imaginée et réalisée qu'en deux dimensions. L'importance de l'espace tri-dimensionnel dans lequel se déroulent les étapes essentielles à la $v^{21}{ }^{21}$ n'a été reconnue que très récemment. Les modifications de la structure spatiale dépendent de facteurs épigénétiques ${ }^{22}$ qui permettent aux organismes d'ajuster cette fonction essentielle au développement, par le biais notamment du contrôle nutritionnel. De nombreuses observations, de la levure à la souris, montrent que la sénescence est associée à une altération de ces mécanismes qui mettent en cause une grande famille de protéines, les Sirtuines $^{23}$, activées par l'activité physique et la restriction calorique (régime équilibré). Au carrefour des voies de I'expression génomique, de la réparation de I'ADN et de la production d'énergie, cette famille explique les bienfaits de la diète et de l'exercice sur le vieillissement.

\section{Instabilité du génome et dysfonction artérielle}

Les cancers et les maladies cardiovasculaires sont les deux premières causes de mortalité en France. L'incidence de ces affections est très directement corrélée à l'âge ; il en est de même pour les affections neurodégénératives comme la maladie d'Alzheimer.

Principale responsable des maladies cardiaques et vasculaires, l'athérosclérose est liée à l'âge en raison d'une modification progressive de la structure des artères. Soumises à des processus de dégradation résultant du contact prolongé des protéines de la paroi artérielle avec le glucose sanguin (glycation) et d'oxydation du cholestérol, elles se rigidifient et favorisent l'apparition de plaques de graisse ${ }^{24}$. La fragmentation des fibres d'élastine qui perdent leur parallélisme, la mort cellulaire avec fibrose de remplacement, la réduction du nombre et de l'activité des cellules souches, la réduction des télomérases endothéliales, la production de molécules inflammatoires s'ajoutent à ces mécanismes.

\footnotetext{
${ }^{21}$ La conformation spatiale d'une molécule aussi complexe que I'ADN joue un rôle biologique capital. Dans les chromosomes du noyau cellulaire, I'ADN existe sous deux formes de chromatine, compactée et non-compactée, dont l'état conditionne l'activité. Ce processus permet d'ajuster la transcription aux variations de l'environnement. De nombreuses études montrent que le vieillissement affecte directement cette conformation chromatinienne (J.M. Sedivy. "Aging by epigenetics. A consequence of chromatin damage ?", Experimental Cell Research, 2008, 314, p. 1909).

${ }_{22}$ L'épigénétique est l'étude des changements héritables de l'expression génétique qui ne sont pas dus à des modifications dans la séquence d'ADN. Par des modifications chimiques (méthylation de I'ADN, acétylation des protéines régulant la transcription), elle module la structure spatiale de la chromatine (K. Bloom. "Towards building a chromosome segregation machine", Nature, 2010, 463, p. 446). Âge et épigénétique ont fait l'objet de plusieurs revues (A. Bird. "Perceptions of epigenetics", Nature, 2007, 447, p. 396. J.M. Sedivy. "Aging by epigenetics. A consequence of chromatin damage ?", Experimental Cell Research, 2008, 314, p. 1909).

${ }^{23}$ Les sirtuines sont considérées comme des agents de longévité (V.D. Longo. "Sirtuins in aging and age-related disease", Cell, 2006, 126, p. 257 ; T. Finkel. "Recent progress in the biology and physiology of sirtuins", Nature, 2009, 460, p. 587). Elles peuvent coordonner de multiples fonctions vitales : réparation de I'ADN, résistance des cellules au stress et régulation circadienne du métabolisme. Elles sont actuellement une cible pharmacologique importante.

${ }^{24}$ JF Toussaint, MP Jacob, L Lagrost, J Chapman. L'Athérosclérose : Physiologie, diagnostics et thérapeutiques, Paris, Masson, 2003
} 
Il est possible que la fréquence du cancer chez le sujet âgé ne fasse que refléter le temps nécessaire à l'accumulation des mutations précancéreuses, par l'accumulation de fragments d'ADN non réparés ou en raison de l'altération progressive des mécanismes de réparation tels que les voies de la protéine $\mathrm{p} 53^{25}$.

On a longtemps pensé qu'il s'agissait d'un lien simple. Vivant plus vieux, nous aurions d'autant plus de chances de faire un infarctus ou un cancer que nous sommes en contact prolongé avec les facteurs de risque communs à ces affections: surpoids, sédentarité, tabac, pollution, accélérant la production d'éléments délétères tels que les radicaux libres. Sans nier le rôle de ces facteurs, la réalité est plus complexe. De multiples autres voies métaboliques sont évoquées telles que celles du glucose, source d'énergie majeure, et de sa régulation. De récentes recherches ont mis en évidence chez la souris, la mouche et le lombric, une voie dépendant de l'insuline dont l'activation accroît la durée de vie. Par ailleurs, l'épuisement des cellules souches pourrait également jouer un rôle.

\section{Prévention}

Pour le moment seules deux options sont validées: la restriction calorique et l'exercice physique régulier. L'activité physique est associée à une augmentation de la longévité et à une réduction de la mortalité cardiovasculaire, de la morbidité cancéreuse et à une amélioration de la santé mentale ${ }^{26}$. L'exercice quotidien modéré a des effets préventifs sur de nombreuses maladies liées à l'âge : manifestations de l'athérosclérose, fibrillation auriculaire, diabète, ostéoporose, cancer, troubles cognitifs. L'évidence repose sur des études portant sur des dizaines de milliers de personnes.

La diversité des effets de l'exercice suggère une régulation située très haut dans la hiérarchie des processus métaboliques, en amont des grands mécanismes régulateurs, au niveau des gènes "maîtres" (master genes). De récents travaux ont mis en cause un groupe de facteurs régulant la transcription de I'ADN, capables à leur tour d'activer toute une série de voies et suggérant un lien fort entre les facteurs contrôlant l'énergie cellulaire et la longévité ${ }^{27}$. L'exercice physique modéré et régulier maintient ainsi la forme physique, l'autonomie - qui en dépend - et la qualité de vie du sujet âgé ; même pour une personne de 80 ans, il est encore capable d'augmenter de deux années la durée de vie.

\footnotetext{
25 T Finkel. "The common biology of cancer and ageing", Nature, 2010, 448, p. 767

${ }^{26}$ Expertise collective Inserm. Activité physique : contextes et effets sur la santé. 2008

${ }^{27}$ AJ Levine. "Coordination and communication between the p53 and IGF-1-AKT-TOR signal transduction pathways", Genes \& Development, 2006, 20, p. 267
} 
Dans le diabète de la maturité, les gènes responsables de la résistance à l'insuline appartiennent à un groupe des gènes dits de stockage ou "économes" (thrifty genes), sélectionné au cours de l'évolution pour permettre aux individus soumis aux incertitudes d'apport alimentaire d'atténuer l'hypoglycémie et ses effets durant les périodes de famine. Ce mécanisme, source de nombreuses maladies métaboliques, montre que ces gènes sont adaptés à un phénotype humain physiquement actif et pauvre en ressources, et non sédentaire et sur-nourri, comme c'est le cas désormais pour la plupart des pays développés.

De fait, la restriction calorique en régime équilibré, c'est-à-dire I'adéquation des apports énergétiques, est l'autre intervention qui, à l'âge adulte, prévient le vieillissement. Elle est connue de longue date et son efficacité est démontrée aussi bien chez le singe, le rat, le poisson, le ver de terre ou la levure que chez I'homme. Son mécanisme d'action implique plusieurs déterminants-clefs en amont des principales voies métaboliques ou dans la gestion des réserves énergétiques, tels que l'insuline, les capteurs de type kinase ou les Sirtuines, ou la p53, mais elle facilite aussi l'autophagie en accélérant l'élimination des organites cellulaires dégradés.

\section{Un programme?}

Existe-t-il un programme génétique de vieillissement ou celui-ci n'est-il simplement que la marque du temps sur nos fonctions les plus courantes et les plus indispensables?

Un programme paraît fort peu probable eu égard à la constante et, à ce jour, infaillible règle de la finitude des constituants. Les cellules de la barrière intestinale ne se maintiennent que quelques heures dans leur fonction de préservation du milieu intérieur et de sélectivité des molécules autorisées à pénétrer l'organisme pour le nourrir (digestion). Les cellules de la barrière cutanée sont abandonnées au milieu extérieur (desquamation) et renouvelées après quelques semaines dans leur fonction de défense et de maintien du contenu en eau et de la température corporelle. Les globules rouges ne persistent que 120 jours dans leur fonction de transport sanguin de l'oxygène. De plus, comme pour l'apoptose cellulaire (seul processus sélectif de mort programmée et non de vieillissement), l'évolution observée au sein de chaque famille taxonomique montre la nécessaire dégradation des éléments constitutifs (individus) au profit de la pérennité des systèmes constitués (l'espèce) rappelant en cela le très ancien constat Platonicien ${ }^{28}$. Si un programme génétique de vieillissement existait, nous aurions déjà découvert des membres de notre espèce ou d'une autre, porteurs de mutations ayant abouti à la suppression de vieillissement, c'est à dire à la perpétuation indéfinie, à l'immortalité. Seule notre imagination nous permet encore de croire à cela.

\footnotetext{
${ }^{28}$ Platon. Le Banquet
} 


\section{Couplages et Pressions Environnementales}

L'apogée des capacités physiques, au moment de la maturité reproductive, et la durée de vie déterminent les caractéristiques du cycle de vie individuel au sein d'une espèce mais l'amélioration, ou la dégradation des conditions environnementales modifie considérablement ces durées respectives (la première démonstration en fut apportée par Steven Austad chez l'opossum ${ }^{29}$ ). La levée des contraintes externes permet ainsi de repousser ces barrières : dans l'espèce humaine (comme pour les espèces domestiquées par l'Homme), la durée de vie s'est considérablement accrue après que la production agricole puis l'élévation des niveaux de consommation énergétique ont, dans les pays développés du moins, soulagé chacun dans sa quête quotidienne d'une ration alimentaire minimale. Chez le primate en captivité, le double avantage d'une nutrition garantie et de l'absence de prédateurs pourrait même favoriser l'expression d'attitudes sociales extrêmement raffinées allant jusqu'à des comportements d'accompagnement aux mourants ${ }^{30}$ très proches des nôtres.

À l'inverse la pression exercée sur nos proies montre un phénomène adaptatif opposé à savoir une diminution de la taille à l'âge adulte et un abaissement de l'âge moyen de la maturité reproductive, associé à une réduction de la durée de vie. Les espèces sauvages des poissons pêchés dans toutes les mers du monde ont, peu ou prou, suivi ces phases avant d'aboutir parfois à l'extinction. On peut penser qu'une ré-élévation des contraintes externes sur l'espèce humaine se traduirait par des reculs équivalents, exprimés par l'élasticité des courbes biométriques de mortalités, de survie, d'espérances de vie et de démographie. Tous ces indicateurs dépendent en effet de l'état d'activation énergétique du système étudié (les ressources dont dispose l'espèce et sa capacité à les exploiter) comme de l'équilibre transitoire entre ce niveau d'énergie et celui des systèmes concurrents, incluant ses prédateurs.

Au delà de nos limites génomiques, les principaux facteurs s'opposant à la poursuite de notre expansion incluent actuellement les virus et les bactéries (avec l'acquisition accélérée de résistances aux traitements), les modifications environnementales (la température n'étant que la première d'une longue liste), la saturation démographique, la disponibilité des ressources (à commencer par l'accès à l'eau potable) et leur acheminement.

\footnotetext{
${ }^{29}$ Les opossums élevés dans une île, isolés mais abrités, vivent en moyenne $25 \%$ plus vieux que leurs congénères continentaux soumis aux nuées de prédateurs. Ils réduisent aussi leur taux de reproduction et présentent des critères de vieillissement beaucoup moins marqués démontrant qu'une diminution de la pression de sélection permet un allongement de l'ensemble du cycle vital.

30 Un article et un film récents de JR Anderson ("Pan thanatology", Current Biology. mai 2010, 20, p. 349) montrent de façon indiscutable les modifications majeures du comportement de chimpanzés en captivité (caresse, soutien, ralentissement, sidération) à la mort d'une des leurs, mère âgée d'un membre du groupe.
} 


\section{Perceptions, perspectives}

La conscience de l'accélération du temps dépend aussi de la durée de vie. Les études de neurophysiologie montre que notre horloge biologique se décale avec le temps, expliquant la fluctuation de nos perceptions, si bien chantée par Serge Reggiani ${ }^{31}$. Mais les personnes présentant une maladie grave, et dont elles savent qu'elle peut limiter leur espérance de vie à court terme, traduisent plus souvent et plus jeunes que les autres cette fuite inéluctable. Kevin Dez, jeune auteur du théâtre contemporain, exprime ainsi ce sentiment : «Il y a longtemps que j'entame le chant de mon cygne ». Pour tout un chacun, ce chant débute au moment même de la conception, mais il s'élève lorsque survient la confrontation, lorsque s'accroît le fardeau du pathologique, lorsque les processus de destruction accélèrent leur rythme, facilité par la précocité de la maladie, génétique ou non.

Plusieurs facteurs affectant la longévité semblent actuellement s'associer et entrainent un ralentissement dans les régions développées (en France pour l'espérance de vie féminine Figure 3, comme au Japon) ou en développement: les reculs majeurs après transition politique (Zimbabwe) ou après récession économique (Russie) ; une dépendance énergétique, de type exponentielle, pour l'ensemble des pays du monde ${ }^{32}$; enfin la dépendance de la mortalité aux dépenses de santé (caractérisée en France par la consommation de soins et de biens médicaux) dont l'accroissement ne cesse de se réduire depuis 50 ans à proportion du ralentissement de croissance de notre PIB.

Il est probable que l'absence de provisionnement des retraites en France, de plusieurs dizaines de milliards d'euros pour les décennies à venir, contribue aussi à une dégradation de certaines situations sanitaires avec impact immédiat sur les plus vulnérables et les plus âgés d'entre nous. Si la récession se prolonge, on peut alors s'attendre à un arrêt brutal de la progression des courbes d'espérance de vie dans l'ensemble des pays concernés.

\footnotetext{
${ }^{31} \mathrm{G}$ Moustaki. Votre fille a vingt ans, Madame... "Chacun de ses vingt ans pour vous a compté double"

${ }^{32}$ A Lauvergeon, MH Jamard. La troisième révolution énergétique. Paris, Plon, 2008
} 


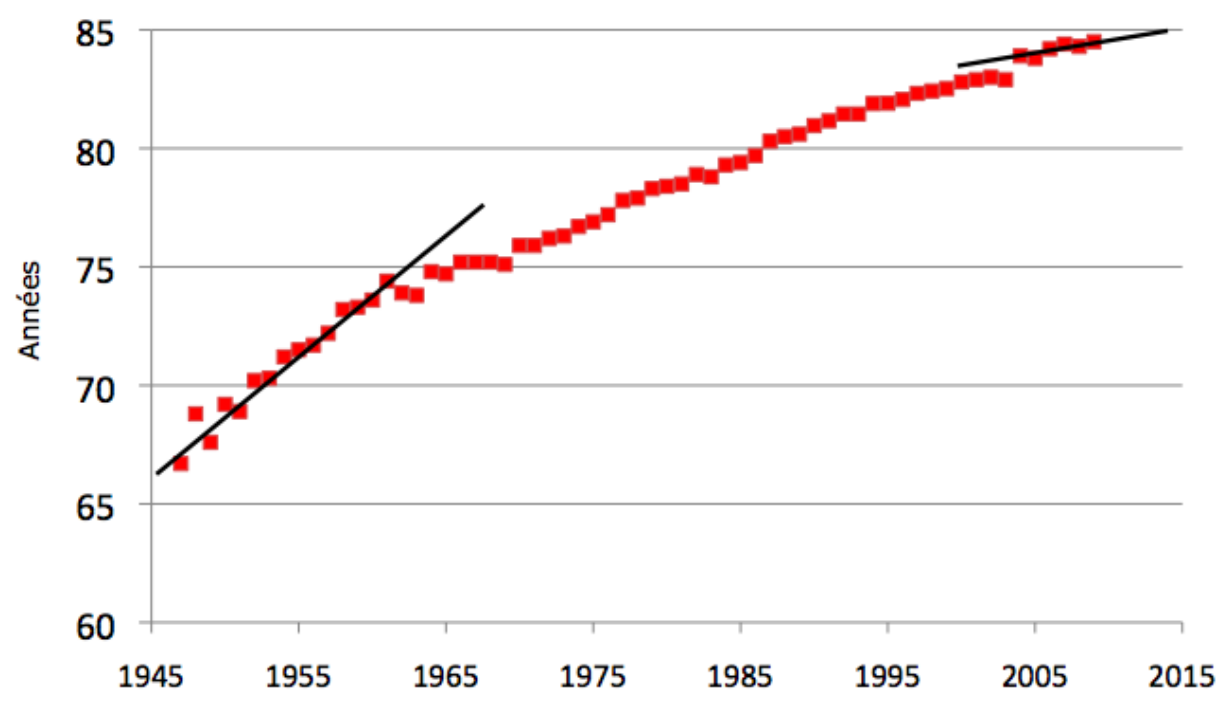

Figure 3 : Évolution de l'espérance de vie féminine en France. Le ralentissement régulier est mesurable par comparaison du gain des dernières années avec celui des années cinquante, permettant une estimation de l'espérance de vie moyenne maximale aux environs de 90 ans. Source : INSEE, Janvier 2010.

Au rayon des espoirs en suspens, on peut aussi ranger notre rêve de bricolage génétique. Le séquençage du génome humain avait pourtant laissé espérer il y a dix ans quelque impact en terme de santé publique. Mais compte tenu de la complexité des régulations en cause et de la très faible capacité des variants communs à expliquer le caractère héritable de pathologies fréquentes ${ }^{33}$ - comme les maladies liées au vieillissement - les éditoriaux récents de Francis Collins, cernant le champ très étroit des applications ayant abouti, et de Craig Venter, appelant à plus de compréhension physiologique des maladies liées au génome humain (c'est-à-dire toutes), ne peuvent tenir lieu de communiqué de victoire ${ }^{34}$.

Quoique révèlent les mécanismes évoqués ici, il semble enfin que nous soyons confrontés à une avalanche explicative à mesure que progresse la connaissance. Chaque source d'interrogation appelle en effet une expérimentation originale mais chacun des résultats de cette recherche nouvelle génère à son tour une multitude de questions. Ainsi nous trouvons nous face à un autre paradoxe, entretenu par l'instabilité de notre cortex et sa quête anxieuse de savoir - dominée par le «que serons-nous demain ? »- qui voit à chaque étape reculer l'horizon de la certitude. La seule permanence reste donc celle de la tentative, de I'illusion, celle de la course sans fin, marquée du sceau de la Reine Rouge de Lewis Carroll ${ }^{35}$.

\footnotetext{
${ }^{33}$ B Jordan. "À la recherche de I'héritabilité perdue", Médecine/Science, 2010, 26, p. 541

34 "The human genome at ten", Nature, 2010, 464, p. 649-674 et 676

35 "It takes all the running you can do, to keep in the same place. If you want to get somewhere else, you must run at least twice as fast". Through the looking-glass and what Alice found there
} 


\section{Conclusion}

La nature du vieillissement biologique est systémique, commune à l'ensemble des espèces, archaïque sur le plan évolutionniste, se confondant avec I'histoire du vivant, et inexorable à l'échelle universelle. Répondre à un processus aussi puissant par trois recettes d'apothicaire, l'activation de deux gènes ou par une molécule, unique et prodigieuse, reste merveilleusement utopique.

L'humanité régresse-t-elle pour autant et saura-t-elle maintenir un certain temps les progrès fulgurants qui furent les siens en terme d'espérance de vie sur les seules dix dernières générations ? Les turbulences actuelles, si elles n'encouragent pas à l'optimisme, sont-elles le signe avant-coureur d'un cycle nouveau ? Rien ne permet de l'affirmer, même si la question se pose avec une acuité de plus en plus claire. Et dans ce cas, répondronsnous à la fascination du naufrage par I'hypnose du nombre, comme nous l'avons fait jusqu'à présent?

Devrions nous, à mesure que progresse notre incompréhension du monde, développer une pédagogie de la limite, défendre l'acceptation pacifique du retour, nous préparer à certaines formes de résignation auxquelles nous convie, contre son gré, Jean Améry ${ }^{36}$, ou que campe Gérard Depardieu dans Mamuth, à l'aube de sa première, et dernière, dérive poétique ? Ou au contraire nous préparer à lutter dans le fracas du reflux pour tenter de préserver d'autres qualités humaines, simples, celles du partage de l'effort, de l'entraide, de l'empathie et de la tolérance vis à vis des faiblesses de l'autre ; lucides et conscients, après Francisco Goya, que les sables du temps engloutissent néanmoins fatalement les lutteurs.

\footnotetext{
${ }^{36}$ J Améry, Du vieillissement : révolte et résignation, Paris, Payot, 1968
} 